\title{
A Study on Compatibility Analysis Based on Minimum Coupling Loss Applicable to Frequency Coordination Combined with Geographic Information
}

\author{
Kyoung-Whoan Suh $^{1}$ and Jeong-Seok Jang ${ }^{2}$ \\ ${ }^{1}$ Electronics Engineering, Kangnam University \\ ${ }^{2}$ Navaid Div. of Telemax Co. \\ ${ }^{1}$ kwsuh@kangnam.ac.kr, ${ }^{2}$ chang1022@hotmail.com
}

\begin{abstract}
With the help of radio propagation prediction of Rec. ITU-R P.1546 combined with geographic information, we present a comprehensive formulation of interference analysis based on the minimum coupling loss, which is applicable to the frequency coordination for wireless systems, and also suggest the S-I plane and local coordinate system for obtaining antenna gains resulting from azimuth and elevation angles between the victim receiver and the interferer. To check the presented methodology, the map with the land-sea mixed zone was taken for the given geographical area of $80 \times 60\left[\mathrm{~km}^{2}\right]$, and computational results were achieved for radar and fixed wireless system in terms of field strength, path profile, BER performance, and protection ratio with maximum allowable interference level. In addition interference powers of the victim receiver have been evaluated as a function of frequency dependent rejection, azimuth and elevation angles of the interferer. The developed methodology of interference analysis in the VHF and UHF bands can be actually applied to assess compatibility of wireless systems regardless of frequency bands as well as applications.
\end{abstract}

Keywords: Antenna pattern, frequency dependent rejection, interference analysis, minimum coupling loss, Rec. ITU-R P.1546

\section{Introduction}

It is well known that the radio spectrum is a vital, but limited natural resource which provides the means to convey audio, video or other information content over distances. Generally VHF/UHF and microwave bands are much preferred due to better propagation characteristics and are getting more spectrum utilities compared with other ones, and these trends are gradually accelerated in commercial and military applications as time goes. So the interference analysis in those bands has been greatly issued to assure the frequency sharing and harmonization between wireless systems [1,2], and the procedure for the frequency coordination should be conducted with interference analysis for the same or adjacent frequency bands. In order to fulfill such a thing with guidelines, the concept of a protection ratio has been used as a generic interference management methodology and criteria. It is defined by a minimum ratio of the relative levels of wanted and unwanted signals at the input 
port of the victim receiver [3, 4]. In principle there are two methodologies to analyze the interference criteria. One is to use Monte Carlo Analysis-SEAMCAT (Spectrum Engineering Advanced Monte Carlo Analysis Tool), which is a statistical methodology for the simulation of random process by randomly taking values from a probability density function [5-7]. The other is the Minimum Coupling Loss (MCL) method, which has been widely used for estimation of interference mechanism even though it is rigid and difficult to implement in many cases not be described in static terms [8]. Therefore recent studies have been presented with reference to radio relay system, fixed satellite and wireless access systems, noncommunication devices into GPS service, and coexistence requirement of LTE networks in view of the frequency coordination as well as interference analysis [9-13].

The transmitted signal inevitably undergoes a path loss involved in radio propagation environments from the transmitter to the receiver. To forecast such propagation loss, Rec. ITU-R P Series provide a lot of propagation models based on the statistical analysis of experimental data or analytical formulations according to frequency ranges relevant to various services [1]. In particular Rec. ITU-R P.1546 provides a method for point-to-area radio propagation predictions for terrestrial services in the VHF and UHF bands, and describes the method for obtaining the field strength as a function of distance combined with geographic information including land, sea, and land-sea paths [14]. Also Guidance on the prediction of point-to-point or point-to-multipoint path loss is provided in Rec. ITU-R P.530 for terrestrial line-of-sight systems [15].

In this paper, we present a comprehensive formulation of interference analysis based on the minimum coupling loss combined with Rec. ITU-R P.1546 and geographic information, which is applicable to the frequency coordination for wireless systems in the VHF/UHF bands, and also suggest the S-I plane and local coordinate system to seek antenna gains resulting from azimuth and elevation angles between the victim receiver and the interferer. To check the proposed methodology, computer simulations are performed for interference analysis between radar and fixed wireless system (FWS) under the assumed parameters and real geographic information. In addition interference effects of the victim receiver are discussed here in terms of interference power, protection ratio (PR), BER performance, and frequency dependent rejection with frequency offset.

\section{Formulation of Signal and Interference}

\subsection{Received Signal and Protection Ratio}

Figure 1 shows a frequency allocation of existing and new services assigned for FWS and radars, for instance, where $f_{0}$ is the center frequency of the system and $\Delta f$ is the frequency offset. Considering a new frequency band of radar2 with the same band of FWS, as illustrated in Figure 1, in advance interference analysis over co- and adjacent bands should be mandatorily conducted whether compatibility between radar2 and FWS as well as radar1 may exist or not. 


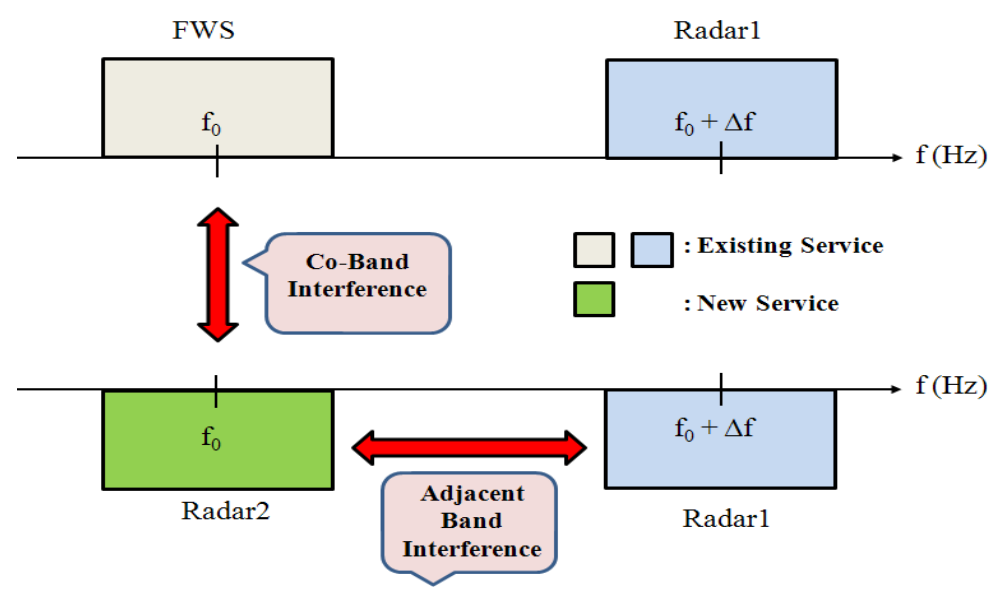

Figure 1. Frequency Bands for Current and New Services

The Rec. ITU-R P.1546 explains a method for point-to-area radio propagation predictions for terrestrial services in the frequency range of $30 \mathrm{MHz}$ to $3000 \mathrm{MHz}$ [14]. It can be used for calculating field strength values over land paths, sea paths and/or mixed land-sea paths between $1.0 \sim 1000 \mathrm{~km}$ for effective transmitting antenna heights, $h_{1}$ less than $3000 \mathrm{~m}$. The propagation curves in this Recommendation means the electric field strength for $1.0 \mathrm{~kW}$ ERP (Effective Radiated Power) at nominal frequencies of 100, 600, and $2000 \mathrm{MHz}$, respectively. For any other frequencies, interpolation or extrapolation of the values obtained for these nominal frequency values should be used to get field strength values by virtue of the methods given in this Recommendation.

For the electric fields combined with the basic transmission loss from Rec. ITU-R P.1546, the received interference power at Rx, $P_{r}$ is given by [16,17]

$$
P_{r}=E_{P .1546}+P_{I}+G_{I}+G_{V}-L_{I}-L_{V}-20 \log _{10} f-139.3-F D R
$$

where $E_{P .1546}$ denotes the field strength $E(d B(\mu V / m))$ for $1.0 \mathrm{~kW}$ ERP, $P_{I}$ is the peak power of interfering system $(\mathrm{dBm}), G_{I}$ is the antenna gain of the interfering system in the direction of the victim receiver $(\mathrm{dBi}), G_{V}$ is the antenna gain of the victim receiver in the direction of the interfering system $(\mathrm{dBi}), L_{I}$ and $L_{V}$ are the insertion losses of interfering system and victim receiver $(\mathrm{dB})$, respectively. And $f$ means the frequency $(\mathrm{MHz})$, and $F D R$ is the frequency dependent rejection (dB) [8], which is given by

$$
F D R(\Delta f)=10 \log _{10}\left[\int_{0}^{\infty} S(f) d f / \int_{0}^{\infty} S(f) R(f+\Delta f) d f\right]
$$

where $S(f)$ is the transmitter power spectral density, $R(f)$ is the receiver selectivity with the receiver tuned to the transmitter frequency, and $\Delta f$ is the tunned transmitter frequency minus the tuned receiver frequency. If the interfering system is operated at co-channel to the victim receiver, the $F D R$ is given by

$$
F D R=\max \left(0,10 \log _{10}\left(B_{I} / B_{V}\right)\right)
$$


where $B_{I}$ is the emission bandwidth of the interfering system and $B_{V}$ is the input bandwidth of the victim receiver.

The degradation of received signal caused by the Gaussian-like multiple interferers, combined with the assumed white Gaussian noise channel, is expressed by $[18,19]$

$$
\begin{gathered}
(C / N)_{t}=[(N / C)+(I / C)]^{-1} \\
(I / C)=\left[\left(I_{1} / C\right)+\left(I_{2} / C\right)+, \ldots,+\left(I_{n} / C\right)\right]
\end{gathered}
$$

where $(N / C)$ is the thermal noise-to-carrier ratio, $(I / C)$ is the equivalent interference-tocarrier ratio, $(C / N)_{t}$ is the total degraded $(C / N)$ due to multiple interferences, and $\left(I_{i} / C\right)(i=1,2, \ldots, n)$ is the i-th interference-to- carrier ratio.

For the frequency coordination over the given link, relating the calculated $(C / I)_{\text {link }}$ with $P R$ including FDR, it can be written by $[16,17]$

$$
(C / I)_{\text {link }} \geq P R=(C / N)_{\min -r q r d}+(N / I)-F D R
$$

where $(C / N)_{\min -r q r d}$ denotes the minimum required carrier-to-noise ratio without interference, and $(N / I)$ is the noise-to-interference ratio which usually gets $10,6,3 \mathrm{~dB}$, depending upon the required services. For instance, taking the maximum allowable interference level of $N / I=6 \mathrm{~dB}$, it brings the degraded $C / N$ of $1.0 \mathrm{~dB}$.

In consequence Figure 2 depicts the concept of $P R$ where $k$ is Boltzman's constant $\left(1.38 \times 10^{-23} \mathrm{~J} / K\right), T$ is Kelvin temperature $(K)$, and $B$ is the receiver bandwidth $(\mathrm{Hz})$.

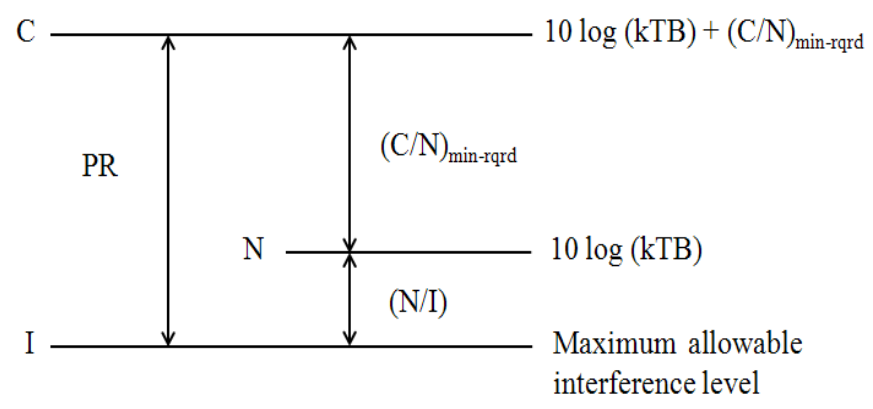

Figure. 2 Concept of Protection Ratio

\subsection{S-I Plane and Local Coordinate System}

Figure 3 shows the geometry of radar and FWS with Tx (Transmitter) and Rx (Receiver) where Rx may be interfered with radar. One may define two vectors, $\vec{S}$ from Rx to Tx and $\vec{I}$ from Rx to radar, and then Signal-Interference (S-I) plane can be construct like Figure 3 with a unit normal vector of $\hat{a}[16,17]$. Information of geographic data for each system entails latitude, longitude, and altitude, and so it can be converted to rectangular coordination system. The discrimination angle $\psi$ can be readily found by the dot product of two vectors like Eq. (7). If FWS and radar use the rotationally symmetric patterns, the receiver antenna gain can be obtained from the antenna pattern corresponding angle $\psi$. 


$$
\cos \psi=\frac{\vec{S} \bullet \vec{I}}{|\vec{S}||\vec{I}|}
$$

Considering the receiver of FWS interfered with potentially multiple interferers such as $I_{1}, I_{2}, \ldots, I_{n}$, each interferer has its own position vector with respect to Rx. Each S-I plane is produced from two vectors $\vec{S}$ and $\vec{I}_{i}$, and consequently the discrimination angle $\psi_{i}$ and its antenna gain $G\left(\psi_{i}\right)$ can be readily found.

On the other hand, consider an arbitrary antenna pattern of the victim receiver, interfered with radar as shown in Figure 4. Then the received interference level depends on the antenna gain, $G(\Delta \varphi, \Delta \theta)$ of $\mathrm{Rx}[20]$, where $\Delta \varphi$ and $\Delta \theta$ are the angle differences between Tx-Rx and Rx-Radar in the azimuth and elevation directions, respectively. Therefore to find these angles, the local coordinate system centred at $\mathrm{Rx}$ is proposed like Figure 4. Assume a point $P_{0}$ located at $1000 \mathrm{~m}$ higher than $\mathrm{Rx}$, but with the same longitude and latitude, and also let this point be $\left(x_{0}, y_{0}, z_{0}\right)$ in the global coordinate system with the origin of the earth center. The vector between $P_{0}$ and Rx can be expressed by

$$
P_{0}-R x=\left(x_{0}, y_{0}, z_{0}\right)-\left(x_{R x}, y_{R x}, z_{R x}\right)=(a, b, c)
$$

where $a, b, c$ are constant and $x_{R x}, y_{R x}, z_{R x}$ are coordinates of $\mathrm{Rx}$ in the global coordinate system.

In order to define Eq. (8) as the $z$ axis in the local coordinate system, one should find the normal vectors $v_{1}, v_{2}$ which are perpendicular to Eq. (8), respectively. If both are retrieved, then one may set $x$ and $y$ axes corresponding to $v_{1}$ and $v_{2}$, respectively. Hence what we want is to find transformation $T$, which is an Affine transformation function, relating $v_{1}, v_{2}$, $v_{3}$ in the global coordinate system to $x, y, z$ in the local coordinate system. Based on the theory of basis change [21], the matrix of transformation $\left[T^{-1}\right]$ is solved by

$$
\left[T^{-1}\right]=\left(v_{1}^{T} v_{2}^{T} v_{3}^{T}\right)=\left(\begin{array}{ccc}
\frac{c}{r_{1}} & \frac{a b}{r_{2}} & \frac{a}{r_{3}} \\
0 & -\frac{a^{2}+c^{2}}{r_{2}} & \frac{b}{r_{3}} \\
-\frac{a}{r_{1}} & -\frac{b c}{r_{2}} & \frac{c}{r_{3}}
\end{array}\right)
$$

Therefore the transformation $\mathrm{T}$ with the origin at $\mathrm{Rx}$ in the local coordinate system is to use $[T]$ from the inverse of $\left[T^{-1}\right]$. For the 3 -dimensional coordinate $r$ in the global coordinate system, it can be expressed by

$$
T(r)=[T]\left(r-R_{X}\right)
$$




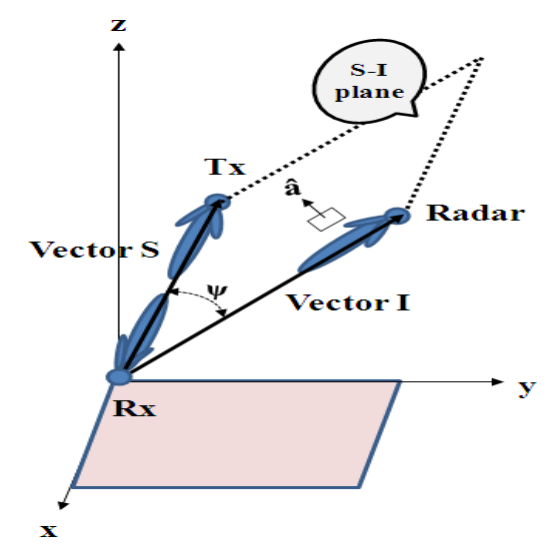

Figure 3. S-I Plane with Signal and Interference Vectors

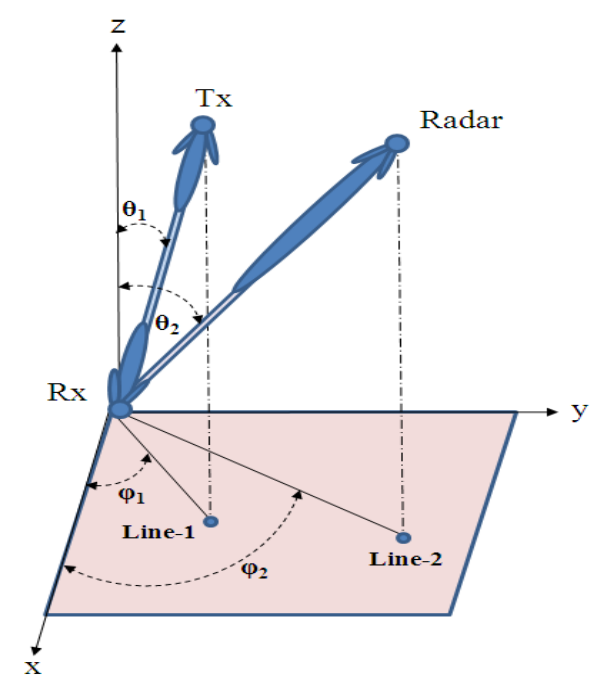

Figure 4. Azimuth and Elevation Angles of Rx to Tx and Radar

\section{Simulation Results and Discussion}

\subsection{Field Strength and FDR}

To show some computational results from the above formulations, real geography information with latitude, longitude, and altitude was adopted for the given the area of $80 \times 60\left[\mathrm{~km}^{2}\right]$ as seen in Figure 5, where $P 1 \sim P 7$ denote the positions along with the path of a warship (radar1). Figure 6 depicts path profiles with the $1^{\text {st }}$ Fresnel zone between $\mathrm{Rx}$ and radars. Moreover Figure 7 illustrates the distribution of field strength $E(d B(\mu V / m))$ around radar1 located at $\mathrm{P} 4$ and radar2, which was calculated for the mixed path (land-sea) by Rec. ITU-R P.1546 for $1.0 \mathrm{~kW}$ ERP. About 34.08 and $68.35 \mathrm{~dB}(\mu \mathrm{V} / \mathrm{m})$ at Rx were obtained from the corresponding transmitters, radar 1 and radar2, respectively.

The assumed FWS characteristics are shown in Table 1, for the simplicity, where FWS means a point-to-point microwave system used for transmitting data or other information, and its operating frequency centers at $2.7 \mathrm{GHz}$ with bandwidth of $40 \mathrm{MHz}$. Then the calculated $P R$ yields $32.3 \mathrm{~dB}$ for the given maximum allowable interference level of $N / I=6.0 \mathrm{~dB}$. In 
order to evaluate interference effect between FWS and radar, Figure 8 illustrates BER versus $C / N$ curves as a function of $N / I=\infty, 10,6,3 \mathrm{~dB}$ for FWS interfered with radar. For the BER curve of $C / I=\infty$, it yields performance in case of no interference.

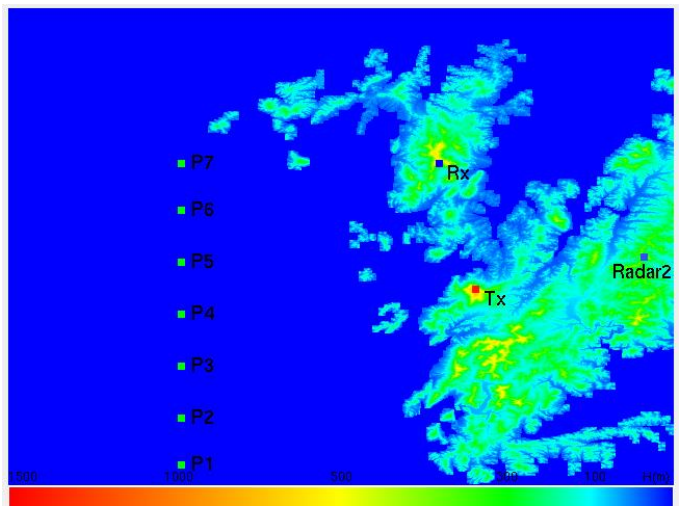

Figure 5. Systems on Real Map
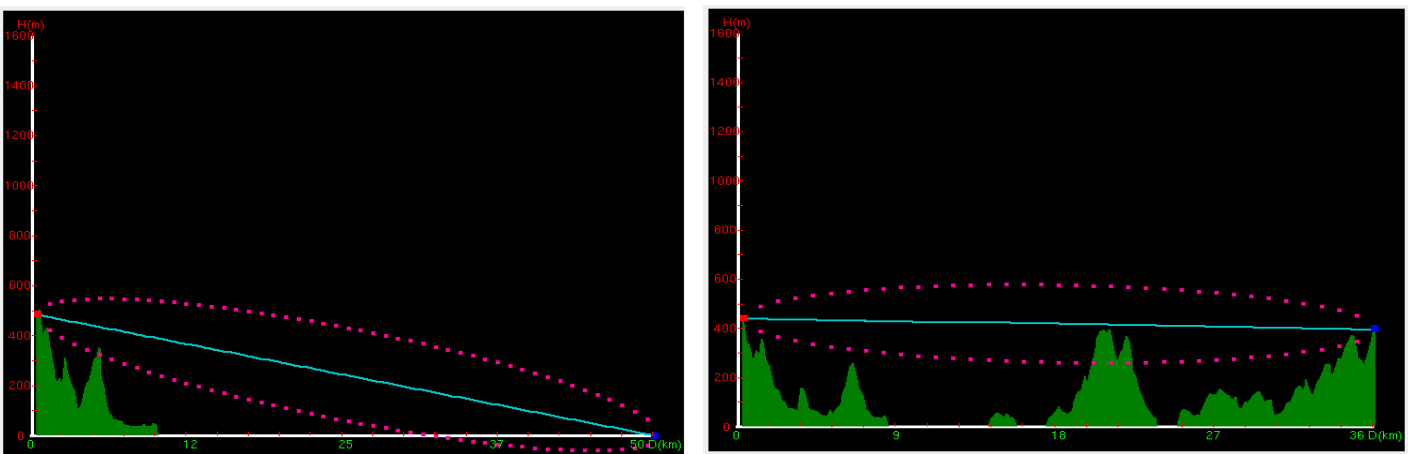

Figure 6. Path Profiles for Rx-Radar1 at P4 (left) and Rx-Radar2 (right)
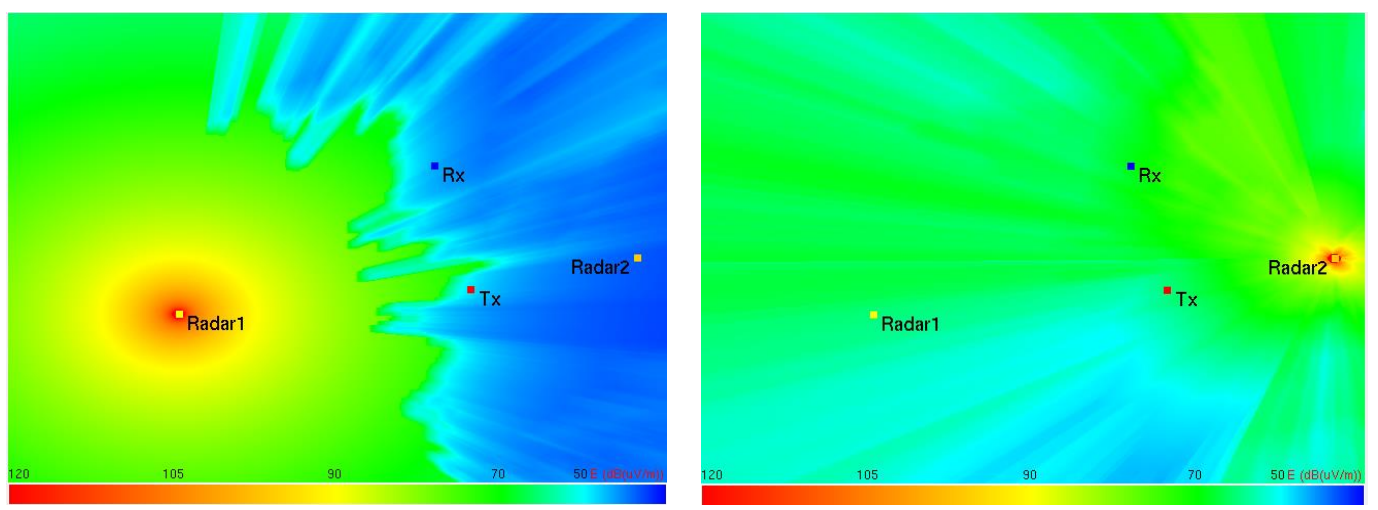

Figure 7. Electric Field Distribution around Radar1 and Radar2 
Table 1. FWS Parameters and $P R$

\begin{tabular}{lll}
\hline Parameters & Values & Remarks \\
\hline Tx power & $27 \mathrm{dBm}$ & Center freq. $=2.7 \mathrm{GHz}$ \\
Ant. gain & $40 \mathrm{dBi}$ & $G_{t}=G_{r}$ \\
$(C / N)_{\text {mrqd }}$ & $26.3 \mathrm{~dB} @$ BER $10^{-6}$ & $64-\mathrm{QAM}$ wo coding \\
$N$ & $-97.98 \mathrm{dBm}$ & $\mathrm{BW}=40 \mathrm{MHz}$ \\
$C$ & $-71.68 \mathrm{dBm}$ & \\
$I$ & $-103.98 \mathrm{dBm}$ & $I / N=-6.0 \mathrm{~dB}$ \\
$P R(=C / I)$ & $+32.3 \mathrm{~dB}$ & $F D R=0 \mathrm{~dB}$ \\
\hline
\end{tabular}

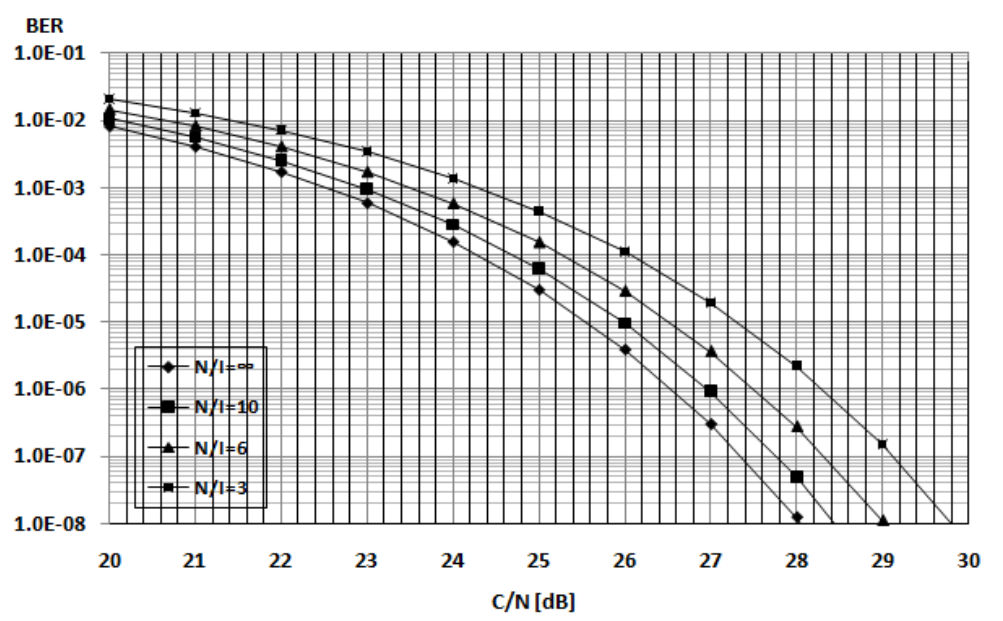

Figure 8. BER Performance for N/I

On the other hand, to check the variation of $P R$ over $F D R$, it was assumed that radar interferes with Rx of FWS. For instance, the power spectral density $S(f)$ of radar1 with the solid line and receiver selectivity $R(f)$ of FWS with the dotted line were taken as shown in Figure 9 [22]. For computing FDR in Eq. (2), the integration was actually done from -45 $\mathrm{MHz}$ to $+45 \mathrm{MHz}$ for the center frequency of FWS because the cumulative power beyond that bandwidth is negligible. Table 2 summarized the minimum required $P R$ of FWS combined with FDR with respect to frequency offset. It is clear that the required $P R$ decreases with increasing frequency offset. On the other hand, the calculated results of FDR and $P R$ for radar 2 were used from Figure 9 and Figure 10 in the reference [16] for the specified spectrum masks [23]. 
Relative power spectral density [dB]

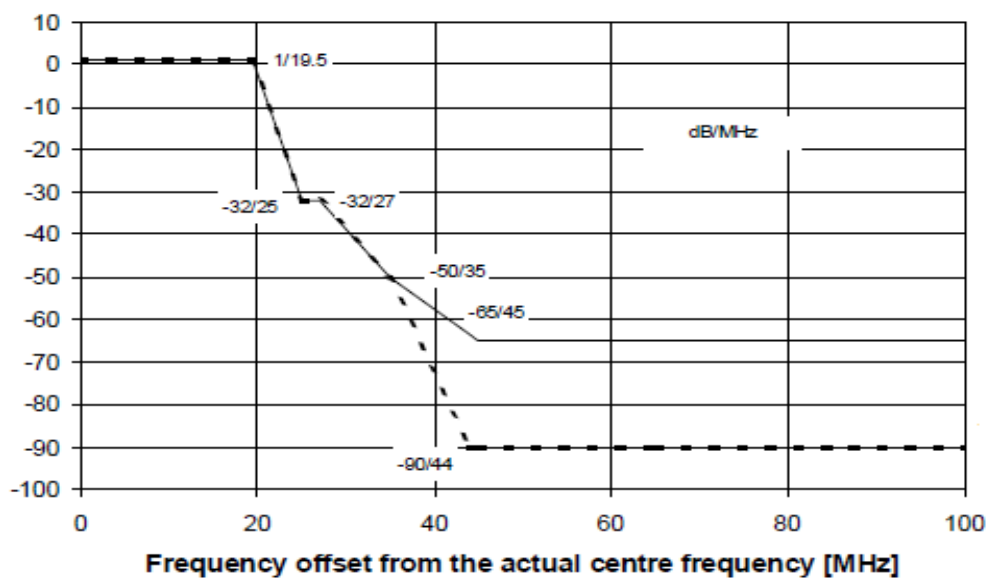

Figure 9. Radar1 Spectrum Mask (solid line) and Receiver Selectivity (dotted line)

Table 2. Required $P R$ of $R x$ for Radar1/2

\begin{tabular}{lllll}
\hline & Radar1 & & Radar2 & \\
$-\Delta f(\mathbf{M H z})$ & $\boldsymbol{F D R}(\mathbf{d B})$ & $\boldsymbol{P R}(\mathbf{d B})$ & $\boldsymbol{F D R}(\mathbf{d B})$ & $\boldsymbol{P R}(\mathbf{d B})$ \\
\hline 0 & 0 & 32.3 & 0 & 32.2 \\
10 & 0.23 & 32.07 & 0 & 32.3 \\
20 & 1.96 & 30.34 & 1.93 & 30.37 \\
30 & 4.86 & 27.44 & 7.81 & 24.49 \\
40 & 17.07 & 15.23 & 42.1 & -9.8 \\
\hline
\end{tabular}

\subsection{Interference Analysis}

First let's consider interference effect of Rx with a rotationally symmetric antenna pattern, while radar is interfering with Rx of FWS. Table 3 illustrates the assumed characteristics of radars. For convenience, the centre frequency of radar and its peak power are $2.7 \mathrm{GHz}$ and 40 $\mathrm{dBm}$, respectively, with system losses $L_{I}=L_{V}=0 \mathrm{~dB}$, and radar is also supposed to be operated at the co-channel of FWS. Then FDR can be readily obtained by $0 \mathrm{~dB}$ due to the same bandwidth. In addition to calculate antenna gains, rotationally symmetrical antenna patterns were adopted for both systems $[23,24]$. But $D / \lambda=18$ was taken for FWS where $D$ is the maximum antenna size and $\lambda$ is the wavelength of frequency.

Table 3. Assumed Radar Parameters

\begin{tabular}{ll}
\hline Parameters & Values \\
\hline Centre frequency & $2.7 \mathrm{GHz}$ \\
Peak power & $40 \mathrm{dBm}(10$ Watts $)$ \\
Main beam gain & $40 \mathrm{dBi}\left(G_{t}=G_{r}\right)$ \\
Pulse width & $0.1 \mu \mathrm{sec}$ \\
Rx IF bandwidth & Radar1/2: About $40 / 28 \mathrm{MHz} @ 3 \mathrm{~dB}$ \\
Pulse repetition rate & $2000 \mathrm{pps}$ \\
Distance from Rx & Radar1/2: $50 \mathrm{~km} / 36.6 \mathrm{~km}$ at P4 \\
Radar altitude & Radar1/2: $487 \mathrm{~m} / 88.6 \mathrm{~m}$ lower than Rx \\
\hline
\end{tabular}


Also with a view to calculating interference power under system parameters, Figure 10 illustrates the system geometry of FWS and radar1 with discrimination angle $\psi_{i}$ resulting from interference and signal vectors. The angle $\psi_{i}$ can be easily obtained by the dot product of two vectors $\vec{S}$ and $\vec{I}_{i}$, based on the S-I plane shown in Figure 3. So interference powers for radar1 positions are shown in Figure 11, where the main beam of radar1 is assumed to be in the direction of Rx. Since interference power at each position is greater than the maximum allowable interference level $(-103.98 \mathrm{dBm})$, radar1 is seriously harmful to Rx.

Figure 12 shows interference powers of Rx as a function of frequency offset and azimuth angle, where the azimuth angle $0^{\circ}$ is set in the direction of radar1 main beam on the S-I plane in Figure 3. From Figure 11 interference power at $\mathrm{P} 4$ yielded the maximum value of about $96.4 \mathrm{dBm}$. The curve of frequency offset $0 \mathrm{MHz}$ corresponds to the co-channel operation, and its curve crossing the maximum allowable interference level is about $4^{\circ}$. Therefore it was shown that all interference powers are less than the maximum allowable interference level, $103.98 \mathrm{dBm}$ only if the azimuth angle gets greater than $4^{\circ}$.

In the similar way, one may expect that interference power of Rx regarding frequency offset and elevation angle is the same result as Figure 12 due to the rotationally symmetric antenna pattern. In consequence to assure compatibility for Rx, radar should have at least the off-axis angle greater than $4^{\circ}$ from the main beam direction, regardless of frequency offset, azimuth and elevation angles.

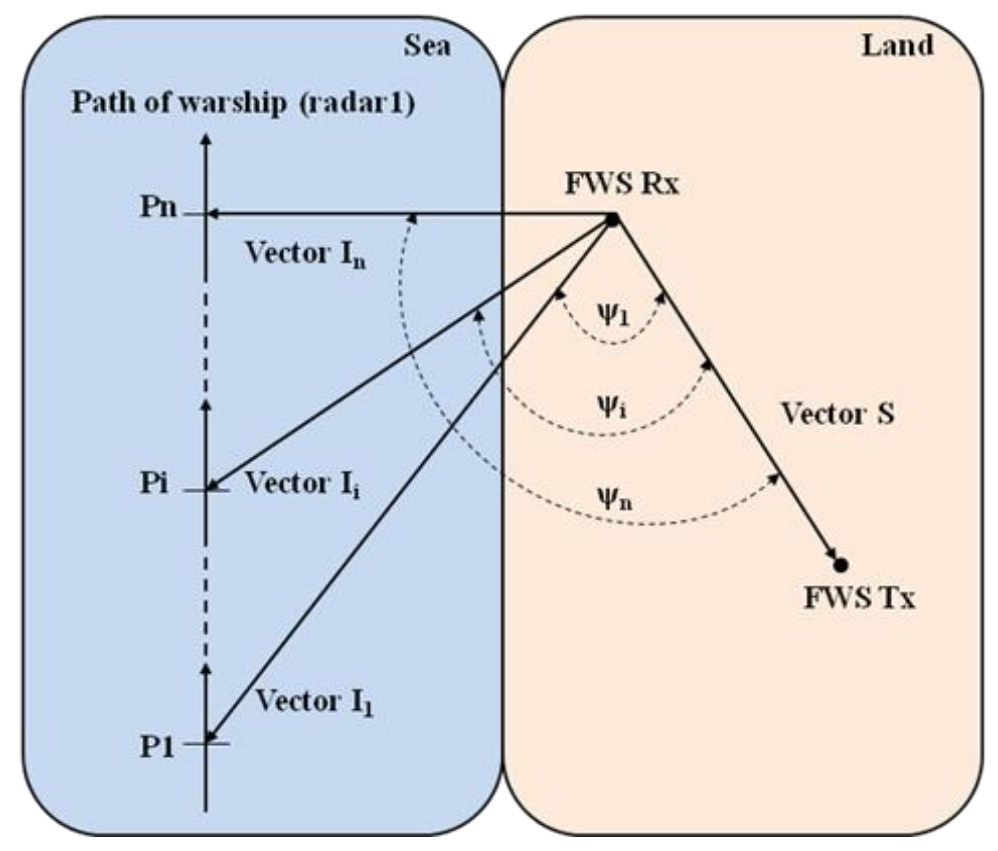

Figure 10. System Geometry of FWS and Radar1 


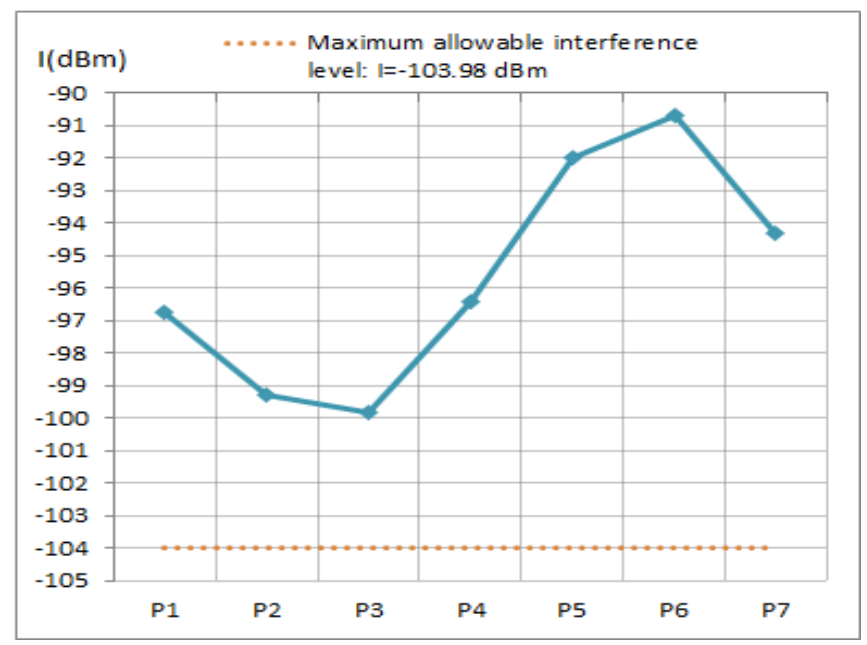

Figure 11. Interference Powers of Rx for Radar1 Positions

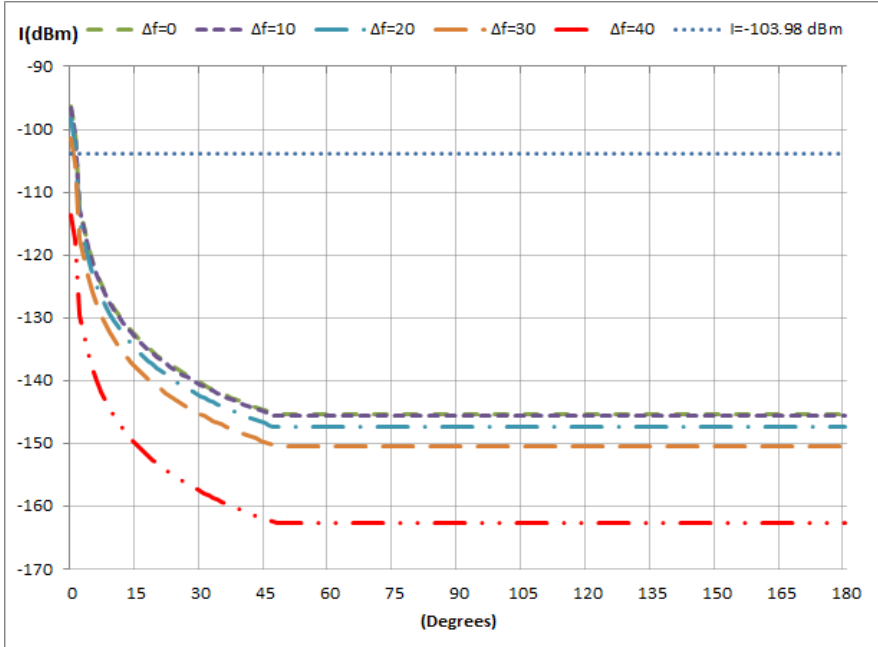

Figure 12. Interference Powers of Rx for Frequency Offset and Azimuth Angle

Next in order to calculate antenna gain and interference power for an arbitrary antenna pattern based on the local coordinate system, S-I planes with multiple interferers located in Figure 5 were depicted in Figure 13. For instance, the antenna patterns of Rx were adopted as the same ones of the mobile base-station as shown in Figure 14, and Table 4 described the antenna types used for systems. Based on the local coordinate system, Table 5 presented Rx antenna gains resulting from elevation and azimuth angles. From these results, Figure 15 illustrated interference powers of $\mathrm{Rx}$ as a function of radar1 position. Compared with Figure 11, all interference powers are much greater than the maximum allowable interference level of $-103.98 \mathrm{dBm}$ due to the different antenna patterns.

Finally Figure 16 presented interference powers of Rx for azimuth angles of radar1 located at P4. The azimuth angle $0^{\circ}$ is set in the direction of radar main beam on the S-I plane in Figure 13. The curve intersects the dotted line of maximum allowable interference level at about $42^{\circ}$. Therefore it was shown that all interference powers are less than the maximum allowable interference level of $-103.98 \mathrm{dBm}$ only if the azimuth angle is greater than $42^{\circ}$, and 
then $\mathrm{Rx}$ will not be affected by radar1. In addition to examine the interference effect of $\mathrm{Rx}$ from radar2 operating at the co-band of FWS, Figure 17 illustrated interference powers of Rx for the variation of azimuth angle. Unlike Figure 16, all interference powers are much greater than the maximum allowable interference level regardless of azimuth angle. Therefore it is concluded that radar2 is severely harmful to Rx of FWS.

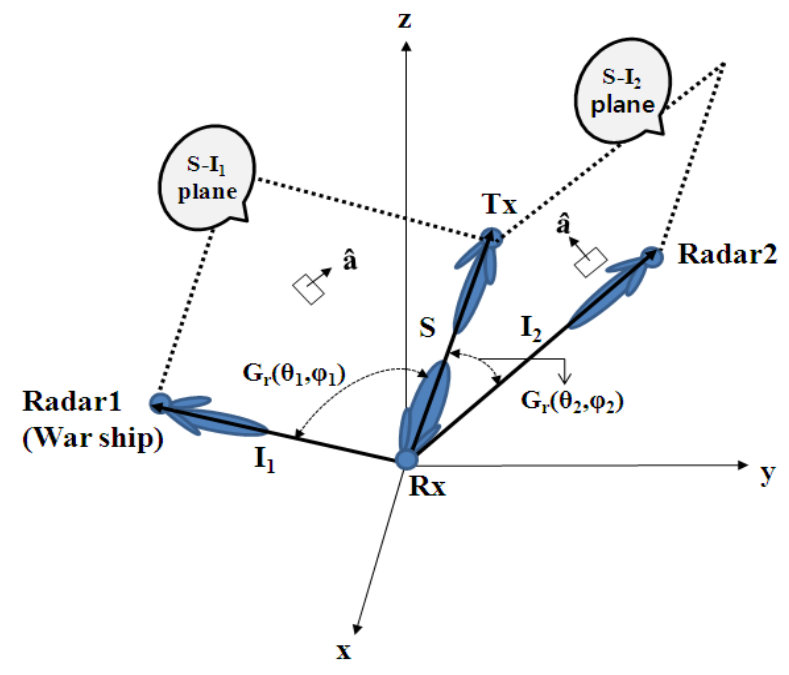

Figure 13. S-I Planes with Multiple Interferers
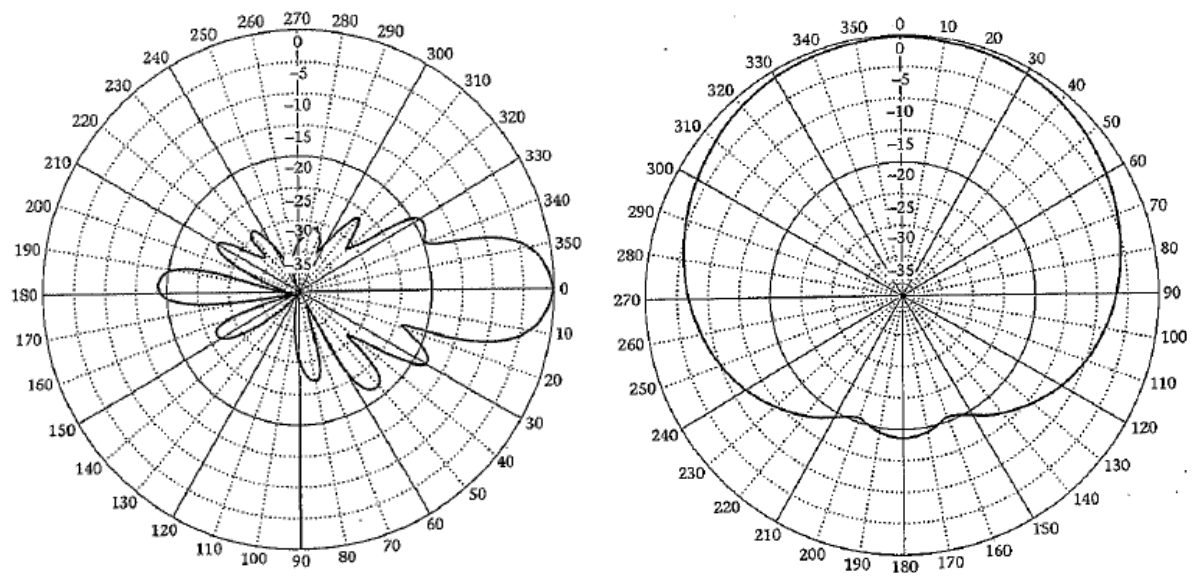

Figure 14. Antenna Patterns of Victim Receiver for Elevation (left) and Azimuth (right)

Table 4. Antenna Characteristics of Systems

\begin{tabular}{|l|l|l|l|}
\hline \multicolumn{2}{|l|}{ Systems } & Antenna patterns & Remarks \\
\hline \multirow{2}{*}{ FWS } & $\mathrm{Rx}$ & Figure 14 & $G_{\max }(\theta, \varphi)=40 \mathrm{dBi}$ directed to Tx \\
\cline { 2 - 4 } & $\mathrm{Tx}$ & Rec. ITU-R F.699 [24] & Rotationally symmetric type, $D / \lambda=18$ \\
\hline \multicolumn{2}{|l|}{ Radar1/2 } & Rec. ITU-R M.1652 [25] & Rotationally symmetric type \\
\hline
\end{tabular}


Table 5. Antenna Gains of Rx Corresponding to Elevation and Azimuth Angles

\begin{tabular}{|c|c|c|c|c|c|c|c|c|}
\hline & \multicolumn{9}{|c|}{ Radar1 positions } & \multirow{2}{*}{ Radar2 } \\
\cline { 2 - 8 } Parameters & P1 & P2 & P3 & P4 & P5 & P6 & P7 & Rarn \\
\hline$\Delta \theta$ & 0.84 & 0.86 & 0.89 & 0.95 & 1.03 & 1.11 & 1.15 & 0.43 \\
\hline$\Delta \varphi$ & 37.28 & 41.39 & 47.46 & 55.84 & 67.52 & 83.21 & 99.99 & 42.99 \\
\hline $\mathrm{G}(\Delta \theta) \mathrm{dB}$ & 0 & 0 & 0 & 0 & 0 & 0 & 0 & 0 \\
\hline $\mathrm{G}(\Delta \varphi) \mathrm{dB}$ & -1.2 & -1.5 & -1.9 & -2.5 & -3.9 & -5.6 & -7.7 & -1.6 \\
\hline $\mathrm{G}(\Delta \theta, \Delta \varphi)$ & -1.2 & -1.5 & -1.9 & -2.5 & -3.9 & -5.6 & -7.7 & -1.6 \\
\hline $40+\mathrm{G}(\Delta \theta, \Delta \varphi)$ & 38.8 & 38.5 & 38.1 & 37.5 & 36.1 & 34.4 & 32.3 & 38.4 \\
\hline
\end{tabular}

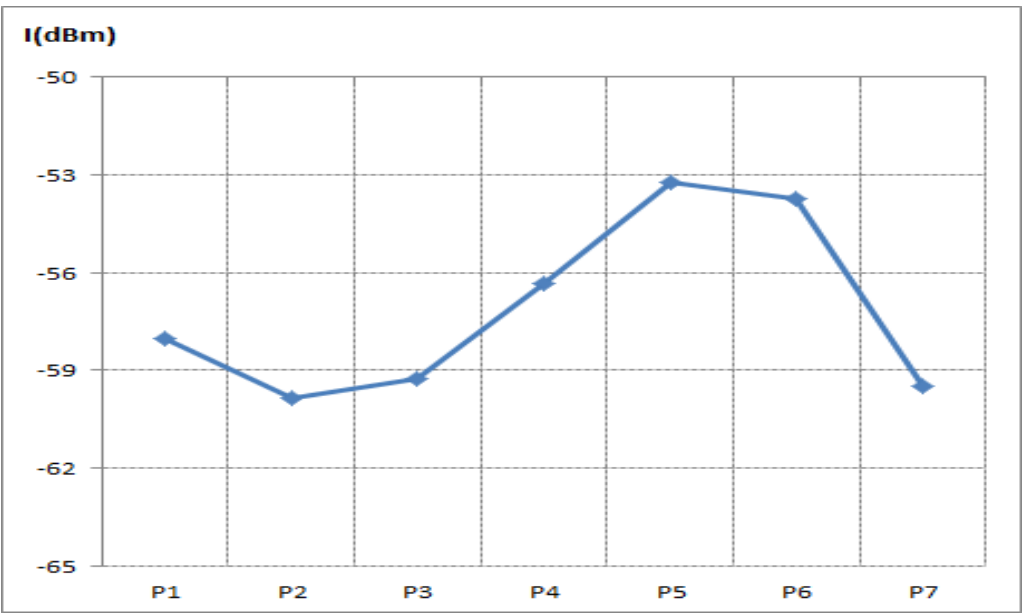

Figure 15. Interference Powers of Rx as a Function of Radar1 Position

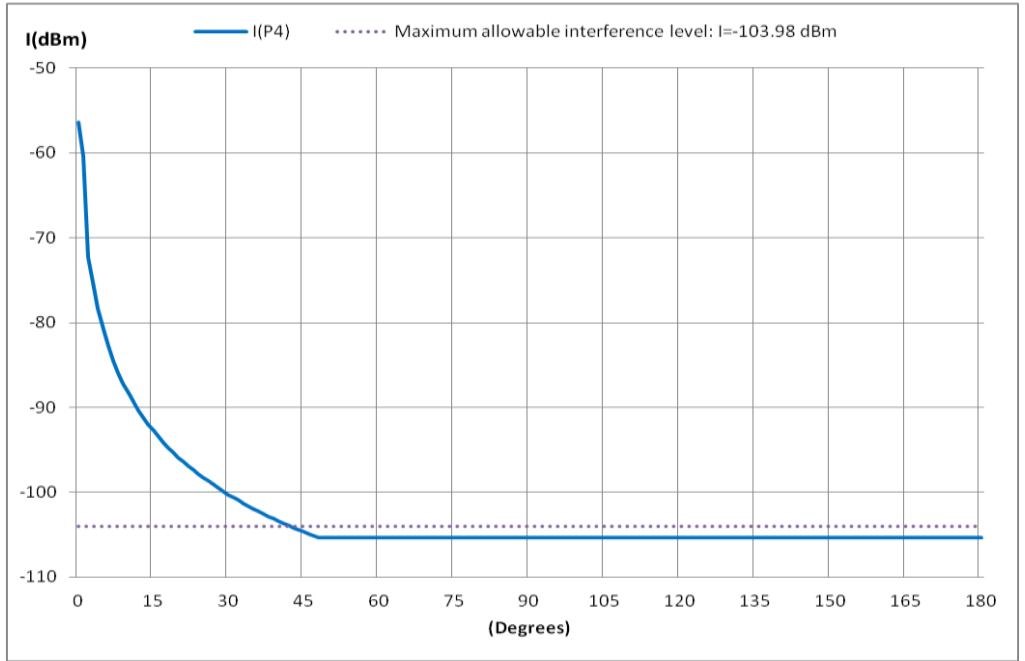

Figure 16. Interference Powers of Rx for Azimuth Angle of Radar1 at P4 


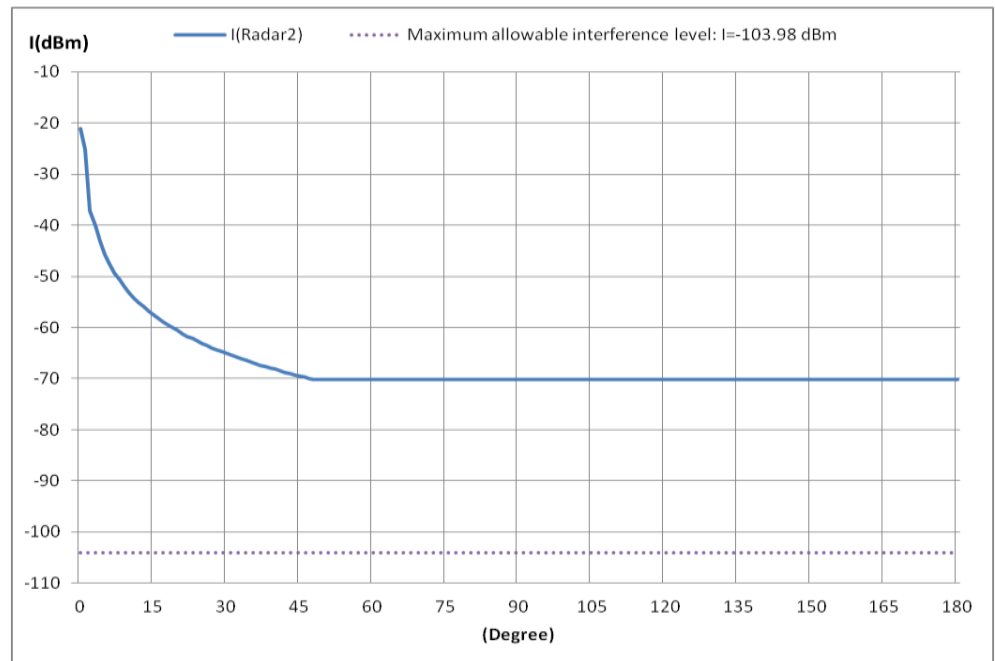

\section{Figure 17. Interference Powers of Rx for Azimuth Angle of Radar2}

\section{Conclusions}

In this paper by using radio propagation prediction of Rec. ITU-R P.1546 combined with geographic information, a comprehensive formulation of interference analysis was presented based on the minimum coupling loss applicable to frequency coordination for wireless systems, and also the S-I plane and local coordinate system were suggested for obtaining antenna gains, depending on azimuth and elevation angles between the victim receiver and the interferer for an arbitrary antenna pattern. To illustrate the presented methodology, the land-sea mixed area was chosen for the given map with $80 \times 60\left[\mathrm{~km}^{2}\right]$ as real geographic information. A variety of numerical results were obtained for radar and fixed wireless system in view of field strength, path profile, BER performance, and protection ratio with maximum allowable interference level. Moreover by introducing S-I plane and local coordination system, interference powers of the victim receiver were readily calculated and discussed as a function of frequency dependent rejection, azimuth and elevation angles of the interferer. The developed methodology of interference analysis in the VHF and UHF bands can be actually applied to assess compatibility of wireless systems operating co- and adjacent bands.

\section{Acknowledgements}

This work was supported by the fund of Agency for Defense Development (ADD) in 2011, Korea.

\section{References}

[1] ITU Radiocommunication Sector (ITU-R), http://www.itu.int/itu-r.

[2] J. D. Laser and J. H. Reed, "Interference rejection in wireless communications", IEEE Communications Magazine, vol. 14, (1997), pp. 37-62.

[3] K. W. Suh, "A generalized formulation of the protection ratio applicable to coordination in digital radio relay networks", Radio Science, vol. 42, RS1007, doi:10.1029/2006RS003470, ( 2007).

[4] N. H. Jeong, J. H. Lee, and K. W. Suh, "Analysis of frequency sharing between mobile systems and radiolocation radars in VHF band", in the 12th International Conference on Advanced Communication Technology (ICACT), (2010), pp. 1179-1183.

[5] ITU, Report ITU-R SM.2048-1, "Monte Carlo simulation methodology for the use in sharing and compatibility studies between different radio services or systems", (2002). 
[6] ITU, Rec. ITU-R M.1634, "Interference protection of terrestrial mobile service systems using Monte Carlo simulation with application to frequency sharing", (2003).

[7] I. K. Cho, I. K. Lee and Y. O. Park, "Study on coexistence between long term evolution and digital broadcasting services", Int'l Journal of Advanced Science and Technology, vol. 38, (2012) January, pp.72-95.

[8] ITU, Rec. ITU-R SM.337, "Frequency and distance separations", (1997).

[9] L. F. Abdulrazak, Z. A. Shamsan and T. Abd. Rahman, "Potential penalty distance between FSS receiver and FWA for Malaysia", WSEAS Transaction on Communications, vol. 7, no. 6, (2008), pp. 637-646.

[10] ITU, Rec. ITU-R M.1461-1, "Procedure for determining the potential for interference between radar operating in the radiodetermination service and systems in other services", (2003).

[11] Y. S. Shim and I. K. Lee, "Interference analysis from non-communication device into GPS service", International Journal of Multimedia and Ubiquitous Engineering, vol. 7, no. 1, (2012), pp. 15-22.

[12] O. Salami, A. Bagula and H. A. Chan, "Evaluation of Interference in Inter-working Multi-hop Wireless Networks", International Journal of Security and Its Applications, vol. 4, no. 4, (2010), pp. 27-42.

[13] A. Oudah, T. A. Rahman and N. Seman, "On the Impact of MIMO antennas on collocation and coexistence requirements of LTE networks in $2.6 \mathrm{GHz}$ frequency band", International Journal of Multimedia and Ubiquitous Engineering, vol. 8, no. 1, (2013), pp.1-10.

[14] ITU, Rec. ITU-R P.1546-4, "Method for point-to-area prediction for terrestrial services in the frequency range $30 \mathrm{MHz}$ to $3000 \mathrm{MHz}$ ", (2009).

[15] ITU, Rec. ITU-R P.530-13, "Propagation data and prediction methods required for the design terrestrial lineof-sights-systems", (2009).

[16] K. W. Suh, et al., "Consideration of interference analysis for wireless systems in VHF/UHF bands with geographic information," Int'l Journal of Hybrid Information Technology, vol. 5, no. 3, (2012), pp. 1-12.

[17] K. W. Suh, et al., "Compatibility analysis for wireless systems in VHF/UHF bands with geographic information," International Journal of Communications, Issue 3, vol. 6, (2012), pp. 128 -135.

[18] A. A. R. Townsend, "Digital line-of-sight radio links: A handbook", Prentice-Hall, Upper Saddle River, N. J., (1988).

[19] ITU, Rec. ITU-R SF.766, "Methods for determining the effects of interference on the performance and the availability of terrestrial radio-relay systems and systems in the fixed satellite service", (1992).

[20] T. A. Milligan, "Modern antenna design, second edition”, 2nd Ed., John Wiley \& Sons, Inc., (2005).

[21] H. Anton, "Elementary linear algebra", John Wiley \& Sons, Inc., (1981).

[22] ETSI EN 301669 1.2.1, "Fixed Radio Systems; Point-to-point equipment; High capacity digital radio systems carrying STM-4 in two $40 \mathrm{MHz}$ channels or $2 \times \mathrm{STM}-1$ in a $40 \mathrm{MHz}$ channel with alternate channel arrangement", (2001).

[23] ETSI EN 301127 1.3.1, "Fixed radio systems; point-to-point equipment; high capacity digital radio systems carrying SDH signals (upto x 2STM-1) in frequency bands with about $30 \mathrm{MHz}$ channel spacing and using copolar arrangements or co-channel dual polarized operation", (2002).

[24] ITU, Rec. ITU-R F.699, "Reference radiation patterns for fixed wireless system antennas for use in coordination studies and interference assessment in the frequency range from $100 \mathrm{MHz}$ to about $70 \mathrm{GHz}$ ", (2004).

[25] ITU, Rec. ITU-R M.1652, "Dynamic frequency selection (DFS) in wireless access systems including radio local area networks for the purpose of protecting the radio determination service in the $5 \mathrm{GHz}$ band", (2003).

\section{Authors}

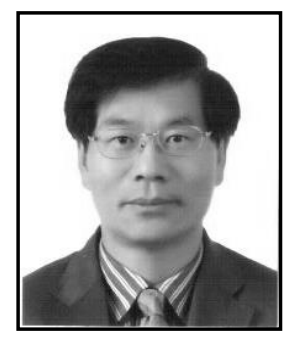

\section{Kyoung-Whoan Suh}

1986.03-1991.08 Ph. D. in Electrical and Electronics Engineering, Korea Advanced Institute of Science and Technology, Korea

1985.03-1988.02 M.S. in Electrical and Electronics Engineering, Korea Advanced Institute of Science and Technology, Korea

1979.03-1983.02 B.S in Electronics Engineering, Kyung-Pook National University, Korea

1999.03-Now Professor, Electronics Engineering, Kangnam University, Korea 
International Journal of Multimedia and Ubiquitous Engineering

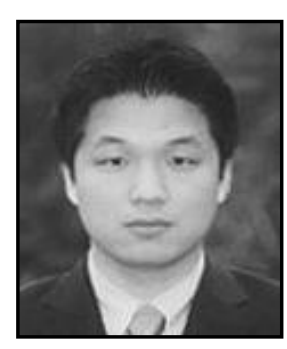

\section{Jeong-Seok Jang}

2008.03-2011.02 Ph. D. in Radio Science and Engineering, Kwangwoon University, Korea

2006.03-2008.02 M.S. in Radio Science and Engineering, Kwangwoon University, Korea

1998.005.02 B.S in Electronics Engineering, Kangnam University, Korea

2011. 03-Now Senior Engineer, Navaid Div. of Telemax Company, Korea 